\title{
Biomarker Guide: The Solution For Distinguish Viral From Bacterial Infections in Patients with Acute Exacerbation of COPD
}

\author{
Salma Messous* \\ Research laboratory, University of Monastir, Tunisia
}

*Corresponding author: Salma Messous, Research laboratory (LR12SP18), University of Monastir, Monastir, Tunisia, Microbiology laboratory, Fattouma Bourguiba university hospital of Monastir, Monastir, Tunisia

Submission: 北 March 19, 2018; Published: 眥 March 22, 2018

\section{Opinion}

Chronic obstructive pulmonary disease (COPD) is an inflammatory disease of the airways, mainly associated with cigarette smoke (CS) exposure. The disease is characterized by a progressive and irreversible decline in lung function caused by airflow obstruction, destruction of parenchyma, and emphysema $[1,2]$.

The pathophysiological changes seen in COPD have been well characterised and are used to diagnose patients. Exposure to inhaled pollutants, primarily cigarette smoke (CS), is thought to lead to the chronic airway inflammation seen in COPD via the activation of structural and inflammatory cells within the lung (epithelial cells and alveolar macrophages). These in turn release chemotactic mediators which recruit additional inflammatory cells (CD8+ T cells, neutrophils, monocytes, and lymphocytes) into the lung perpetuating a state of chronic inflammation, which is thought to cause the structural changes in the airway, airway obstruction, and respiratory symptoms [3].

Several inflammatory cells and their mediators participate in the inflammatory response in COPD. Exposure to cigarette smoke, noxious particles, or gases can activate an inflammatory cascade in the airways resulting in the production of a number of potent cytokines and chemokines which play a critical role in the induction of chronic inflammation and subsequent tissue destruction [4]. The current objectives of COPD treatment are to reduce symptoms, and to prevent and reduce the number of exacerbations. While current treatments achieve these goals to a certain extent, preventing the decline in lung function is not currently achievable [5].

Different biomarkers that express the early pan-airway inflammation of the respiratory tract may be useful to distinguish between AECOPD of bacterial, viral or non-infectious origin and could orientate the use of antibiotics at the early stage of hospitalization. These inflammatory biomarkers appear to be increased in the serum of patients with AECOPD. C-reactive protein (CRP) is currently the best studied biomarker in this setting. It is an acute-phase reactant secreted by the liver in response to infection, inflammation or tissue damage with well-documented sensitivity, and is commonly used for diagnosing infectious and inflammatory conditions, including in AECOPD [6].

In contrast, multiplex PCR assays that combine several individual targets in a single reaction are able to differentiate viral and bacterial respiratory infections and to identify co-infections in a time-saving and cost-effectiveness manner $[7,8]$. They are suitable for optimizing the use of antimicrobial drugs, which contributes to reduce the duration of hospital stay and to save money and antibiotic resistance [9]. In this context, we investigated prospectively a cohort of Tunisian patients hospitalized at the emergency room for AECOPD with two specific objectives: firstly evaluating the prevalence of different pathogens associated to these episodes by combining conventional bacterial quantitative culture and multiplex viral and bacterial qPCR assays in sputum specimens, and secondly determining whether CRP values and clinical outcome could be influenced by the type of microbial agent(s) recovered from these samples. The final aim of the study was to propose an algorithm for optimizing the antimicrobial treatment in AECOPD patients at the early stage of hospitalization.

\section{References}

1. Rabe KF, Hurd S, Anzueto A, Barnes PJ, Buist SA, et al. (2007) Global strategy for the diagnosis, management, and prevention of chronic obstructive pulmonary disease: GOLD executive summary. Am J Respir Crit Care Med 176(6): 532-555.

2. Macnee $W$ (2007) Pathogenesis of chronic obstructive pulmonary disease. Clin Chest Med 28(3): 479-513.

3. Stockley RA, Mannino D, Barnes PJ (2009) Burden and pathogenesis of chronic obstructive pulmonary disease. Proc Am Thorac Soc 6(6): 524526 .

4. Barnes PJ, Shapiro SD, Pauwels RA (2003) Chronic obstructive pulmonary disease: molecular and cellular mechanisms. Eur Respir J 22(4): 672-688.

5. Caramori G, Adcock IM, Di Stefano A, Chung KF (2014) Cytokine 
inhibition in the treatment of COPD. Int J Chron Obstruct Pulmon Dis 9: 397-412.

6. Pepys MB, Hirschfield GM (2003) C-reactive protein: a critical update. J Clin Invest 111(12): 1805-1812.

7. Templeton KE, Scheltinga SA, Van den Eeden WC, Graffelman WA Van den Broek PJ, et al. (2005) Improved diagnosis of the etiology of community-acquired pneumonia with real-time polymerase chain reaction. Clin Infect Dis 41(3): 345-351.

8. Perotin JM, Dury S, Renois F, Deslee G, Wolak A, et al. (2013) Detection of multiple viral and bacterial infections in acute exacerbation of chronic obstructive pulmonary disease: a pilot prospective study. J Med Virol 85(5): 866-873.

9. Pozzetto B, Grattard F, Pillet S (2010) Multiplex PCR theranostics of severe respiratory infections. Expert Rev Anti Infect Ther 8(3): 251-253.

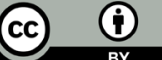

Creative Commons Attribution 4.0 International License

For possible submissions Click Here

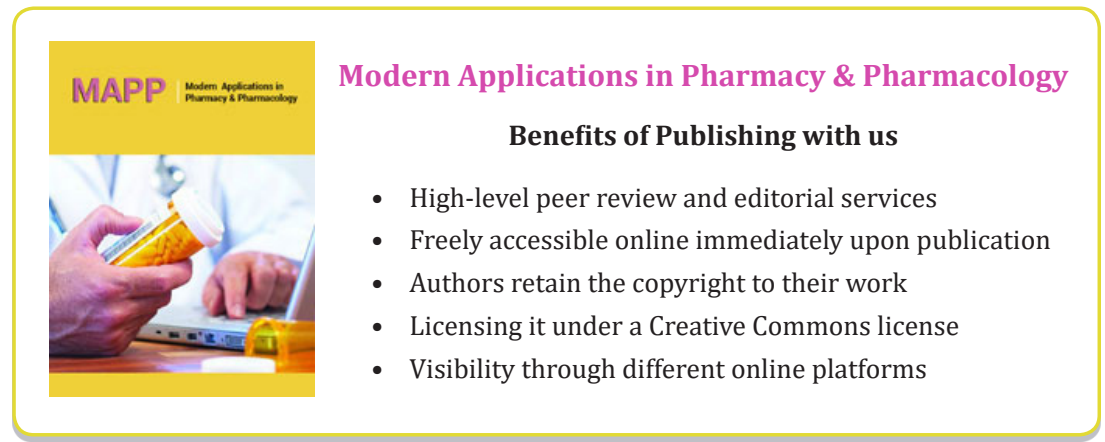

